American Journal of Environmental Sciences 4 (5): 512-521, 2008

ISSN 1553-345X

(C) 2008 Science Publications

\title{
Simulation of Wind Field in Tehran Using Hybrid Diagnostic and Prognostic Models
}

\author{
${ }^{1}$ M. Shafie-Pour, ${ }^{2}$ Y. Rashidi and ${ }^{3} \mathrm{M}$. Ardestani \\ ${ }^{1}$ Faculty of Environment, University of Tehran, Iran \\ ${ }^{2}$ Air Quality Control Company, Municipality of Tehran, Iran \\ ${ }^{3}$ Faculty of Environment, University of Tehran, Iran
}

\begin{abstract}
This article concentrates on Wind Field Simulation in a mega city such as Tehran which is home to nearly 10 million inhabitants. The necessity of having a comprehensive knowledge about the wind field in a certain zone is significant for different reasons; the most important of all would be surveying on the emission and dispersion of pollutants. The wind models can be classified as: Dynamic and Kinematics. In this article the authors have developed a Kinematics model based on Continuity Equation. The final version of the equation being solved is a elliptic partial differential equation. The lateral boundary Conditions are first kind and those for top and bottom are the second kind. In order to initializing the model, the data gathered by two meteorological towers set up in Tehran and also the data of the upper layer atmosphere from Mehrabad Airport have been used. The result of the wind field simulation reveals when the velocity of the synoptic scale wind is low, the condition of the wind flow is entirely affected by the local system of mountain-valley. During the day, the flow is towards the valley to the mountains, while at night it is from the mountain to the valley. The local systems of wind circulation such as mountain-valley and land-sea are closed systems that trigger in removing of the pollutants, their accumulation and their chemical changes in a definite area. It should be noted that wind field simulation, by means of diagnostic (Kinematic) models, depend entirely on the existing data and considering the time we cannot forecast wind field over the observed data. The results of wind field simulation using combination of diagnostic and prognostic (Dynamic) models are significantly improved.
\end{abstract}

Key words: Wind field simulation, continuity equation, mass consistence

\section{INTRODUCTION}

The world of today, due to the population boost and more luxurious way of life, faces a real increasing demand for energy consumption. That is why the renewable energies (solar, wind and water) have come to attention as potential sources of energy for future. Different countries all over the world are now thinking over profiting from these sources of energy. Primarily considerations of economical gains from energy produced by wind turbines, makes research on wind fields even more essential. For instance, the price variation of produced energy in a spot where the average wind velocity is $6 \mathrm{~m} \mathrm{~s}^{-1}$ compared with a spot where it is $8 \mathrm{~m} \mathrm{~s}^{-1}$, exceeds 100 percent. Identifying the wind field in a zone is not limited to wind energy considerations and some other additional actions are influenced by wind field as follows:

- emission and dispersion of air pollutants in the atmosphere
- issues related to the aerodynamic of buildings including stress and pressure distribution, heat loses and ventilation

- effects on transportation, such as location of airports, aerodynamic of automobiles and so forth

- agricultural considerations such as soil erosion as well as the water vaporization and growth of plants

In order to simulate wind field in a particular zone, the existing models are divided into two groups: Prognostic (Dynamic) and Diagnostic (Kinematics). The diagnostic models themselves follow two simulation attitudes: simplified solutions of steady state condition of equations of motion (such as linear techniques) and purposeful analysis of meteorological information considering the physical constrains (e.g., the Mass consistence). Some instances of such models are co: MASCON (1); MSFD (2); NLMSFD (3); WASP (4); NUATMOS (5); WINDS (6); WOCSS (7).

Corresponding Author: M. Ardestani, Faculty of Environment, University of Tehran, Iran 
The dynamic models are established on solving the hydrodynamic and energy equations which are timedependent and written for the atmosphere. The thorough solution of these equations is quite demanding and costly. Moreover, these kind of mentioned models require reliable input, to get benefited from the advantages of the model, which we most of the time lack them. Some instances could be: MEMO (8); MM5 (9); RAMS (10).

The relative simplicity of diagnostic wind models triggered in deploying them in a lot of practical usage; since they do not require a huge input parameters and they are proportionally simpler and regarding the cost of calculations more economical. In fact, these models make use of the existing data quite simply and with high practicality and, by providing some physical conditions, provide the wind field.

\section{GOVERNING EQUATIONS}

The Mass Consistence model (based on conservation of mass) is being used for simulating the wind field in Tehran. This model is based on non compressible form of continuity equation:

$$
\nabla . \boldsymbol{u}=0 \quad \text { in } \Omega
$$

Now the sum of the squares of difference between the wind fields is calculated $\boldsymbol{u}(u, v, w)$ and the observed wind field $\boldsymbol{u}_{0}\left(u_{0}, v_{0}, w_{0}\right)$ will be written in the specified area $\Omega$ as described below:

$$
\begin{aligned}
& E(u, v, w) \\
& =\int_{\Omega}\left[\begin{array}{l}
\alpha_{1}{ }^{2}\left(\left(u-u_{0}\right)^{2}+\left(v-v_{0}\right)^{2}\right) \\
+\alpha_{2}{ }^{2}\left(w-w_{0}\right)^{2}
\end{array}\right] d V
\end{aligned}
$$

$\alpha_{i}(i=1,2)$ are fixed and are considered as the function of stability conditions of the atmosphere( the correcting ratios of horizontal and vertical directions) in horizontal directions and these ratios are considered equal. Here, the aim is to minimize equation based on the conditions of equation, which results in the following equation:

$$
\begin{aligned}
& J(u, v, w ; \lambda) \\
& =\int_{V}\left\{\begin{array}{l}
\alpha_{1}^{2}\left(\left(u-u_{0}\right)^{2}+\left(v-v_{0}\right)^{2}\right)+\alpha_{2}^{2} \\
\left(w-w_{0}\right)^{2}+\lambda\left(\frac{\partial u}{\partial x}+\frac{\partial v}{\partial y}+\frac{\partial w}{\partial z}\right)
\end{array}\right\} d V
\end{aligned}
$$

in which $\lambda=\lambda(x, y, z)$ is Lagrange multiplayer. The Euler-Lagrange equations in accordance with equation are as follows:

$$
\begin{aligned}
& u=u_{0}+\frac{1}{2 \alpha_{1}^{2}} \frac{\partial \lambda}{\partial x}, \\
& v=v_{0}+\frac{1}{2 \alpha_{1}^{2}} \frac{\partial \lambda}{\partial y}, \\
& w=w_{0}+\frac{1}{2 \alpha_{2}^{2}} \frac{\partial \lambda}{\partial z}
\end{aligned}
$$

$\lambda$ can be defined from (1) and (4) supposing that $\alpha_{i}$ at the length of the studied zone remain fixed. Therefore, the following elliptical partial differential equation for $\lambda$ is:

$$
\begin{aligned}
& \frac{\partial^{2} \lambda}{\partial x^{2}}+\frac{\partial^{2} \lambda}{\partial y^{2}}+\left(\frac{\alpha_{1}}{\alpha_{2}}\right)^{2} \frac{\partial^{2} \lambda}{\partial z^{2}} \\
& =-2 \alpha_{1}\left(\frac{\partial u_{0}}{\partial x}+\frac{\partial v_{0}}{\partial y}+\frac{\partial w_{0}}{\partial z}\right)
\end{aligned}
$$

or:

$$
\begin{aligned}
& \frac{\partial^{2} \lambda}{\partial x^{2}}+\frac{\partial^{2} \lambda}{\partial y^{2}}+\frac{\alpha_{v}}{\alpha_{h}} \frac{\partial^{2} \lambda}{\partial z^{2}} \\
& =-\frac{1}{\alpha_{h}}\left(\frac{\partial u_{0}}{\partial x}+\frac{\partial v_{0}}{\partial y}+\frac{\partial w_{0}}{\partial z}\right)
\end{aligned}
$$

in which $\alpha_{h}=1 / 2 \alpha_{1}^{2}, \alpha_{v}=1 / 2 \alpha_{2}^{2}$ and $\quad \boldsymbol{\alpha}=\left(\alpha_{h}, \alpha_{h}, \alpha_{v}\right)$. Boundary conditions which are considered for equation (6) are as:

- $\lambda=0$ or Dirichlet Boundary Condition equal to zero in Lateral Boundaries of the selected zone

- $n \cdot \boldsymbol{\alpha} \nabla \lambda=-n \cdot \boldsymbol{u}_{0}$ or Neumann Boundary Condition above and bellow the zone

For simulating the wind field in the selected zone or solving the equation (6), in order to simplify the topographic effects, terrain following Coordinate system is used as described below:

$$
\begin{aligned}
& X=x, Y=y \\
& Z=\frac{z-h}{H-h}=\frac{z-h}{\pi}
\end{aligned}
$$




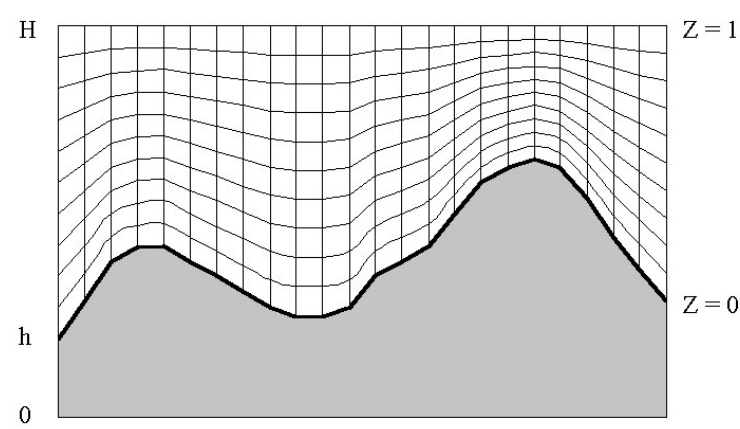

Fig. 1a: Terrain following coordinate system according to the to topography and equation (8)

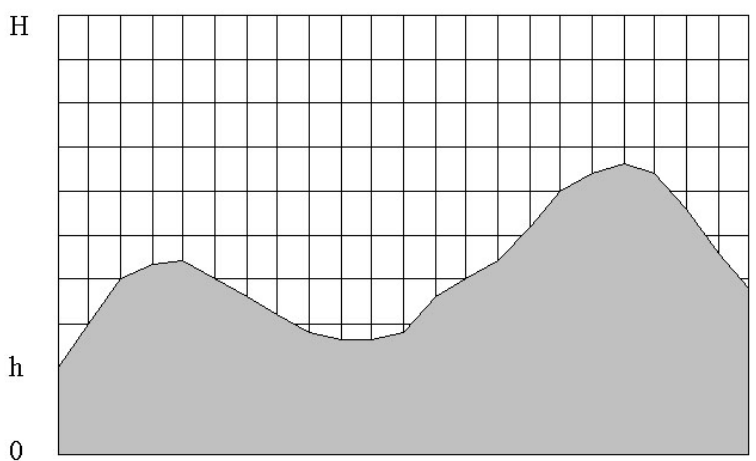

Fig. 1b: Ordinary cartesian coordinates system

in which $(x, y, z)$ are the first Cartesian Coordinates system, $h$ stands for the topography height of each point and $H$ reveals the highest point. The distinction between these two coordinate systems is displayed in Fig. 1. As a result, terrain $Z=0$ is adjusted to the surface of the earth $(z=h(x, y))$ and the terrain $Z=1$ is adjusted to the top boundary which is the fix height $H$.

According to the new defined coordinates, the equation (6) will change form. There are two possible ways for applying these changes. In the first method, the relations of coordinates transformation in continuum mechanics may be used, including covariant differentiation and Christoffel symbol, whereas in the second method the coordinates are directly changing by change of variables. By applying the coordinates transformation the equation (6) will be modified as below:

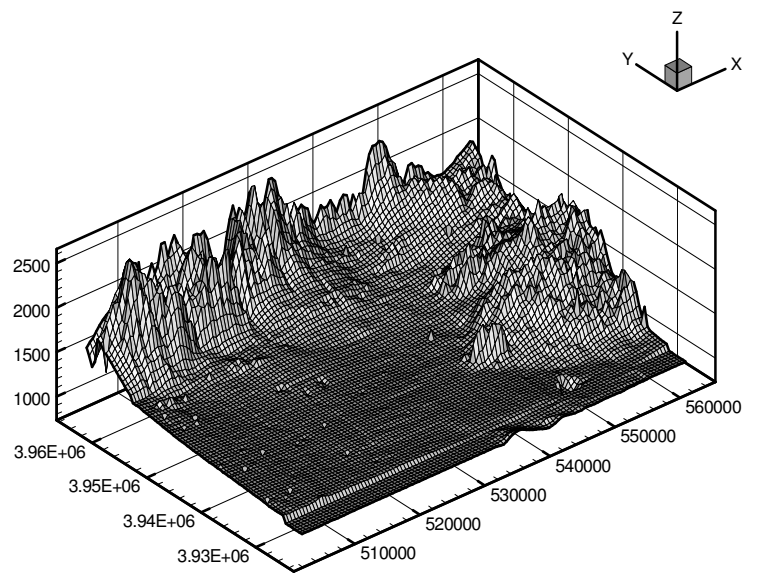

Fig. 2: Tehran topography

$$
\begin{aligned}
& \frac{\partial^{2} \lambda}{\partial X^{2}}+\frac{\partial^{2} \lambda}{\partial Y^{2}}+\left[\left(\frac{Z-1}{\pi}\right)^{2}\left(\begin{array}{l}
\left(\frac{\partial h}{\partial x}\right)^{2} \\
+\left(\frac{\partial h}{\partial y}\right)^{2}
\end{array}\right)+\frac{1}{\pi^{2}} \frac{\alpha_{v}}{\alpha_{h}}\right] \frac{\partial^{2} \lambda}{\partial Z^{2}} \\
& +2\left(\frac{Z-1}{\pi}\right) \frac{\partial h}{\partial x} \frac{\partial^{2} \lambda}{\partial X \partial Z}+2\left(\frac{Z-1}{\pi}\right) \frac{\partial h}{\partial y} \frac{\partial^{2} \lambda}{\partial Y \partial Z} \\
& \left.\left(\frac{Z-1}{\pi}\right)\left[\begin{array}{l}
\frac{\partial^{2} h}{\partial x^{2}}+\frac{\partial^{2} h}{\partial x^{2}}+\frac{2}{\pi^{2}} \\
\left(\left(\frac{\partial h}{\partial x}\right)^{2}+\left(\frac{\partial h}{\partial y}\right)^{2}\right)
\end{array}\right]\right) \frac{\partial \lambda}{\partial Z} \\
& =-\frac{1}{\alpha_{h}}\left[\begin{array}{l}
\frac{\partial u_{0}}{\partial X}+\frac{\partial v_{0}}{\partial X}+\left(\frac{Z-1}{\pi}\right) \\
\left(\frac{\partial u_{0}}{\partial Z} \frac{\partial h}{\partial x}+\frac{\partial v_{0}}{\partial Z} \frac{\partial h}{\partial y}\right)+\frac{1}{\pi} \frac{\partial w_{0}}{\partial Z}
\end{array}\right]
\end{aligned}
$$

Equation (8) is an elliptical equation in general, in these boundary conditions in terrain following coordinate system, they are stated as below:

$\lambda=0$ on the lateral boundaries

$\frac{\partial \lambda}{\partial Z}=0$ on the top terrain

$$
\frac{\partial \lambda}{\partial Z}=\frac{\pi / \alpha_{h}\left[\begin{array}{l}
\left(u_{0}+(\partial \lambda / \partial X)\right) \partial h / \partial x+ \\
\left(v_{0}+(\partial \lambda / \partial Y)\right) \partial h / \partial y-w_{0}
\end{array}\right]}{\left[(\partial h / \partial x)^{2}+(\partial h / \partial y)^{2}\right]+\alpha_{v} / \alpha_{h}}
$$

on the earth terrain 
Am. J. Environ. Sci., 4 (5): 512-521, 2008

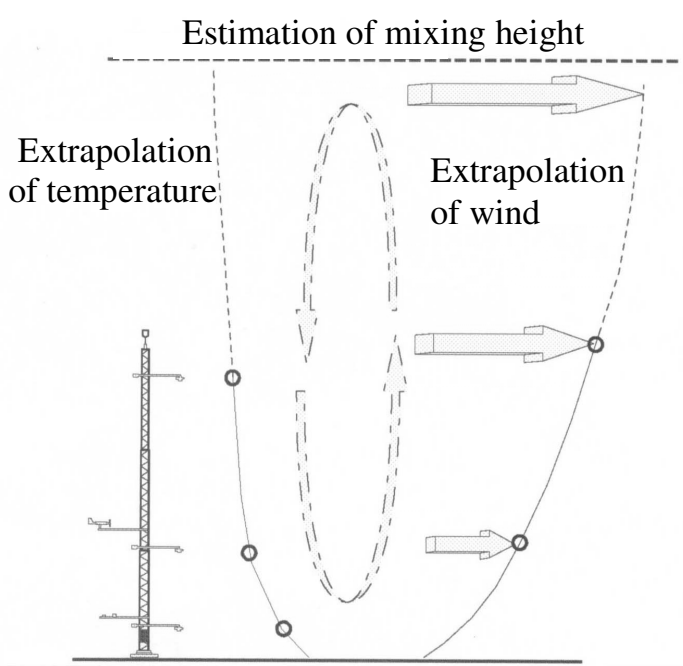

Fig. 3: Meteorological tower in atmospheric boundary layer

\section{PREPARING THE INITIAL WIND FIELD}

In order to solve equation (6), one needs to determine the first wind field $\boldsymbol{u}_{0}\left(u_{0}, v_{0}, w_{0}\right)$ in different heights, all over the selected zone. To prepare the necessary data on the situation of wind pattern in Tehran regarding the topography (Fig. 2) and the effects cause by that, two meteorological towers have been set up in two different points of the city. The first tower is located in Tehransar area $(\mathrm{x}=518325, \mathrm{y}=$ 3955262) UTM Coordinates and the second one in Resalat area $(\mathrm{x}=541717, \mathrm{y}=3955262)$ UTM Coordinates. Both of the towers are 24 meters in height and are equipped with measuring sensors of profiles of temperature, velocity and the wind direction. Besides, the mentioned towers demonstrate the condition of the boundary layer of atmosphere and define its features. Figure 3 represents the mentioned towers substitution in the boundary layer of atmosphere.

In order to determine the first wind field it is quite necessary to utilize the data of the mentioned towers and those collected from the top atmosphere in Mehrabad Airport.

The horizontal and vertical interpolation will also be carried out in this respect. The towers data to the height of surface layer will be extrapolated according to the Monin-Obukhov theory.

The extrapolation of velocity in the vertical direction in the superficial layer of atmosphere is accomplished by the use of a relation proposed by Holstlag (11):

\begin{tabular}{|c|c|}
\hline Turning angle, $\mathrm{D}(\mathrm{h})(\mathrm{deg})$. & Monin-obukhov legth (m) \\
\hline 12 & -30 \\
\hline 10 & -100 \\
\hline 9 & -370 \\
\hline 12 & $10^{4}$ \\
\hline 18 & 350 \\
\hline 28 & 130 \\
\hline 35 & 60 \\
\hline 38 & 20 \\
\hline 39 & 2 \\
\hline
\end{tabular}

$$
u_{z}=\frac{u_{*}}{k}\left\{\ln \left(\frac{z}{z_{0}}\right)-\psi_{m}\left(\frac{z}{L}\right)\right\}, z_{0} \leq z \leq z_{s l}
$$

in which $u_{*}$ is Friction Velocity, $k$ is von karman constant, $L$ is Monin-Obukhov length (demonstrating the Stability Conditions of Atmosphere : stable $L>0$, unstable $L<0$ and neutral $L=\infty), z_{0}$ reveals the surface roughness and $z_{s l}$ stands for the thickness of surface layer $\psi_{m}$ is determined based on the atmospheric stability conditions:

$$
\left.\begin{array}{l}
\psi_{m}(z / L)=\ln \left[\left(\frac{1+x}{2}\right)^{2}\left(\frac{1+x^{2}}{2}\right)\right] \\
-2 \arctan x+\pi / 2, z / L<0 \\
x=(1-16 z / L)^{1 / 4}, \psi_{m}(z / L)=0, z / L=0 \\
\Psi_{m}=-\left[\left(1+\frac{2 a}{3} \frac{z}{L}\right)^{3 / 2}+b\left(\frac{z}{L}-\frac{c}{d}\right)\right], z / L>0 \\
a=1, b=0.667, c=5, d=0.35
\end{array}\right]
$$

The length scale quantity of Monin-Obukhov and Friction Velocity can be determined according to the measurements of the wind field profiles and temperature made on the towers. In addition, the quantity of convectional heat transfer from the surface of the earth to the atmosphere will also be defined. Besides, along with extrapolation in the surface layer, the turning angle of the wind will be corrected according to the following relation and Table 1:

$$
D(z) / D(h)=d_{1}\left[1-\exp \left(-d_{2} z / h\right]\right.
$$


in which $D(z)$ shows the rotating angle at height $z, D(h)$ represents the rotating angle at the reference height (200m) and $d_{1}=1.58, d_{2}=1.0$ are experimental constants. In order to determine the thickness of the surface layer, one ought to define the thickness of the boundary layer of the atmosphere. After defining the thickness of the boundary layer of the atmosphere, the thickness of surface layer is calculated as $z_{s l}=0.1 z_{p b l}$. For the stable conditions of the atmosphere $(L>0)$, it can be noted (13):

$$
z_{p b l}=0.4 \sqrt{\frac{u_{*}}{f} L}
$$

in equation $f=2 \Omega \sin \phi$ is called Coriolis Parameter ( $\Omega$ is the angular velocity of the earth and $\phi$ shows the latitude of the selected zone) the same relation for neutral and unstable conditions (14) are as below $(L<0)$ :

$$
z_{p b l}=0.3\left(\frac{u_{*}}{f}\right)
$$

In the height over the surface layer, the initial wind field, regarding the data of the top of the surface layer and the information of the upper layer atmosphere data at Mehrabad Airport, is determined according to the following relation:

$$
\begin{aligned}
& u_{0}(z)=\rho(z) u_{0}^{u p p e r}, \\
& (z)+(1-\rho(z)) u_{0}^{s l}(\infty) \\
& \rho(z)=\frac{z-z_{s l}}{H-z_{s l}}
\end{aligned}
$$

in the equation(17) $u_{0}^{s l}(\infty)$ is the velocity of the wind at the top of the surface layer and $u_{0}^{\text {upper }}(z)$ represents the data related to the upper layer atmosphere measured by means of radio sound.

In order to make horizontal interpolation in the entire selected zone, the data of several points in which the wind fields are defined, the following relation is often used:

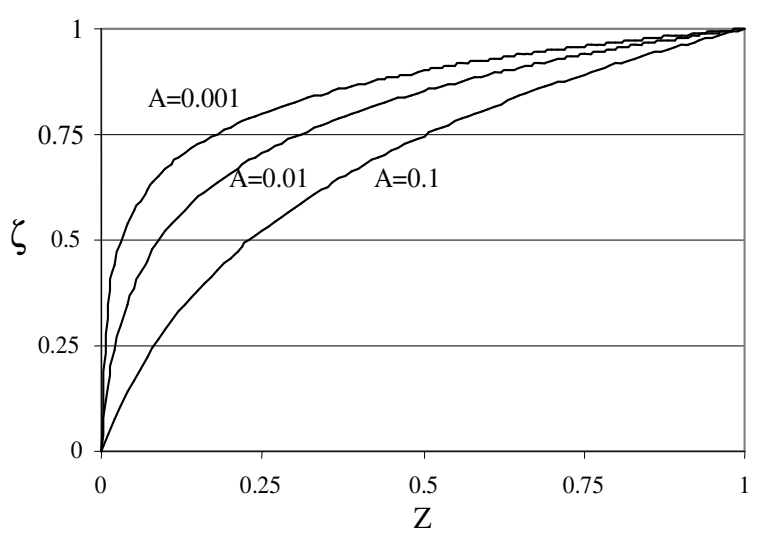

Fig. 4:Effect of parameter A on grid distance near the surface

$$
\left(u_{0}, v_{0}\right)=\frac{\sum_{j=1}^{N}(u, v)^{j} f\left(r_{j}\right)}{\sum_{j=1}^{N} f\left(r_{j}\right)}
$$

in which $(u, v)^{j},\left(u_{0}, v_{0}\right)$ respectively display component of $x$ and $y$ of the wind which should be identified and the vector of velocity in $j$ point. $r_{j}$ is the distance between the point in which the velocity field of the wind is already determined $\left\{(u, v)^{j}\right\}$ and the point where the velocity field $(u, v)^{\circ}$ should be defined. $f\left(r_{j}\right)$ is also a weighting function. The most common weighting function used in this case isas follows:

$$
f\left(r_{j}\right)=\left(\frac{1}{r_{j}}\right)^{2}
$$

It should be mentioned that, it is necessary that the vertical distance between the points near the earth be less, changing vertical coordinates for considering this effect, the following relation can be used:

$$
\zeta=\frac{\ln (1+Z / A)}{\ln (1+1 / A)}
$$

The amount of $A$ is an optional quantity; by adjusting this quantity, the vertical distance between the points near the earth can be changed. Figure 4 displays the effect of this quantity. As represented in the figure, 
the small amounts of $A$ results in the shortening of the vertical distance between the points near the earth.

According to equation (20), the following differentiations should be replaced in the equation (8) and (11):

$$
\begin{aligned}
& \lambda_{Z}=\frac{d \zeta}{d Z} \lambda_{\zeta} \\
& \lambda_{X Z}=\frac{d \zeta}{d Z} \lambda_{X \zeta} \\
& \lambda_{Y Z}=\frac{d \zeta}{d Z} \lambda_{Y \zeta} \\
& \lambda_{Y Z}=\left(\frac{d \zeta}{d Z}\right)^{2} \lambda_{\zeta \zeta}+\frac{d^{2} \zeta}{d Z^{2}} \lambda_{\zeta}
\end{aligned}
$$

\section{NUMERICAL SOLUTION METHOD}

SOR method is one of the iteration methods, being used for solving the elliptical equations. The overall form of the finite difference equation, which is considered for this method (here shown for the 2dimensional condition) is as follows:

$$
\begin{aligned}
& P \Phi_{i k}+W \Phi_{i-1, k}+E \Phi_{i+1, k} \\
& +N \Phi_{i, k+1}+S \Phi_{i, k-1}=r_{i, k}
\end{aligned}
$$

in the last equation $P, W, E, N$ and $S$ are coefficients in finite difference form.The last equation can be appropriately written as bellow:

$$
\begin{aligned}
& \Phi_{i, k}^{(r+1)}=\Phi_{i, k}^{(r)}+\frac{\omega}{P}\left(P \Phi_{i, k}^{(r)}+W \Phi_{i-1, k}^{(r+1)}\right. \\
& \left.+E \Phi_{i+1, k}^{(r)}+N \Phi_{i, k+1}^{(r)}+S \Phi_{i, k-1}^{(r+1)}-r_{i, k}\right) \\
& i=1,2, \ldots . . I, \quad k=1,2, \ldots . . K
\end{aligned}
$$

The effect of the neighboring points which are placed in $(i, k-1)$ and $(i-1, k)$ points are determined by means of the quantity $\Phi$ which has been calculated in $(r+1)$ iteration step, the recursive state of the equation (23) does not make vectorized form to by common SOR method. With a little modification of the equation (23), one can write (Red Black SOR):

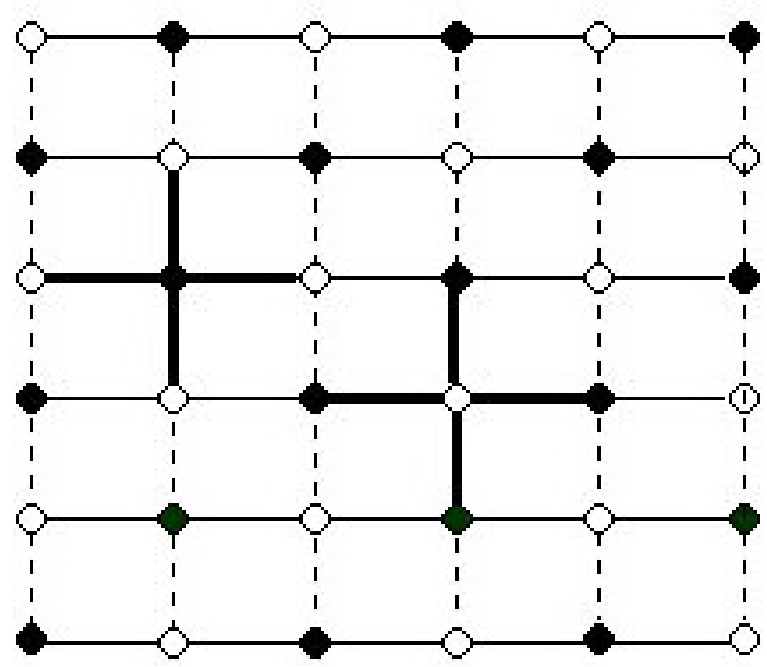

Fig. 5: Displays the modified SOR method

$$
\begin{aligned}
& \Phi_{i, k}^{(r+1)}=\Phi_{i, k}^{(r)}+\frac{\omega}{P}\left(P \Phi_{i, k}^{(r)}+W \Phi_{i-1, k}^{(r)}\right. \\
& \left.+E \Phi_{i+1, k}^{(r)}+N \Phi_{i, k+1}^{(r)}+S \Phi_{i, k-1}^{(r)}-r_{i, k}\right) \\
& i=1,2, \ldots . . I, \quad k=1,2, \ldots . . K
\end{aligned}
$$

Now it can be benefited from the advantage of the last equation:

Provided that $\Phi_{i, k}$ can be define, it would be placed in black or red points (Fig. 5), it is quite easy to determine that unknown variables in the red points only depend on the quantities of the neighboring black points and vice versa.

As a result, in the iteration step $(r+1)$, the new initial quantities in the red points are calculated parallel with calculated values in the black points. This method is repeated the same way until desired convergence is achieved.

\section{THE WIND FIELD SIMULATION IN TEHRAN}

Tehran is located in the longitude of $35^{\circ}, 31$ northern and the latitude of $51^{\circ}, 19^{\circ}$ eastern, which is featured by the slope continued from south to north. Albourz Mountains standing on the north of the city is stretched with a height of 3500 meters in the east direction and 4000 meters in the west direction. The distinction between the high points of Tehran, in the center of the city and Mehrabad Airport (1200 meters above the sea level) and the northern part of Tajrish (1500 meters above the sea level), is 300 meters. 
Am. J. Environ. Sci., 4 (5): 512-521, 2008

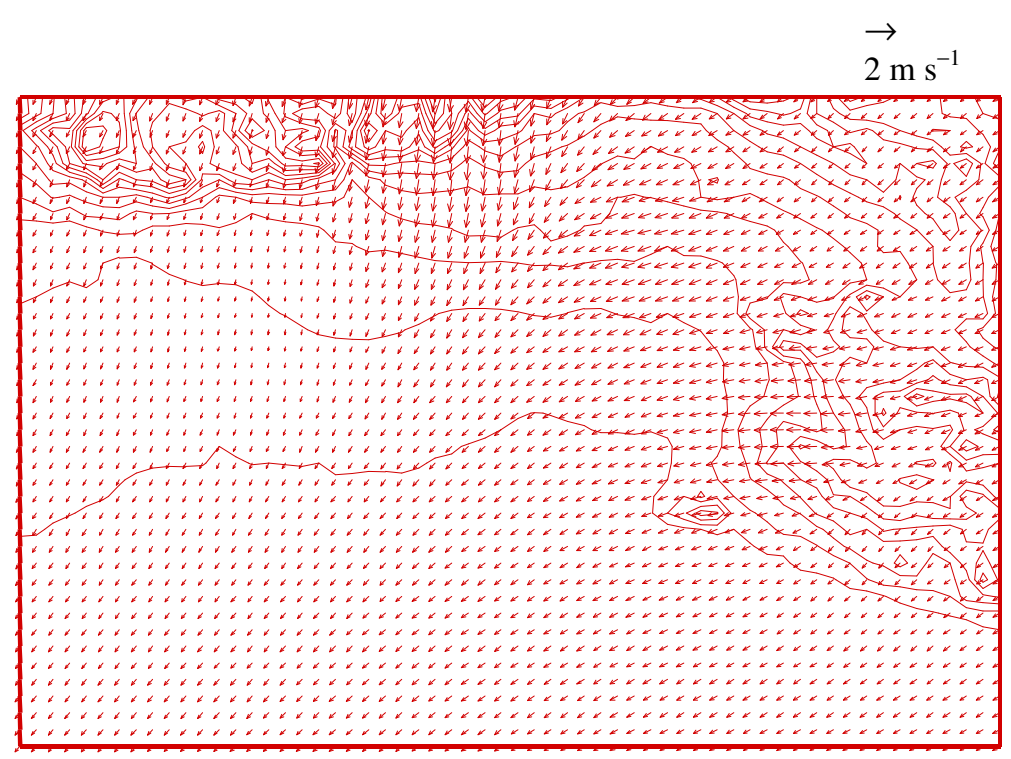

Fig. 6: Wind field simulation in Tehran on 2.9.2001 at 0 GMT at the height of 10 meters

Tehran is sat in the southern part of the Albourz Mountains, which is granted by the step weather. Tehran city is surrounded by these mountains from the north and east direction and is expectedly influenced by a complex wind flow pattern. The industrial zones make up the western part of the city; besides, Tehran Refinery is built in the south of the city. Since the Synoptic wind of Tehran is flowed from the west direction to the east, most of the pollution of this zone is transferred to the center of the city.

The studied area is $59 \times 39.5 \mathrm{Km}$ in the coordinates $(503500,3925000)$ to $(562500,3964000)$. In order to simulate the wind field, the grid size in the horizontal direction is fixed and is considered as $\Delta x=\Delta y=1 \mathrm{Km}$. In the vertical direction, by selecting terrain the following coordinate system and introducing new variable mentioned in equation (20), choosing $A=0.005$ and $\Delta \zeta=0.1$, the simulation is accomplished. The top level of the simulation $(H)$ is considered 4000 meters. Moreover, the quantities of $\alpha_{1}$ and $\alpha_{2}$ have been determined based on the stability conditions of the atmosphere in a way that $\alpha_{1}=1.0$ and $1 / \alpha_{2}$, in turn, allocate the quantities of $0.2,0.5,1.0,2.0,5.0$ to themselves under the conditions of highly unstable, somehow unstable, neutral, somehow stable and highly stable. The procedure of the simulation is as follows:
- determining the initial wind field according to the contents of the second part

- the numerical solution of the model (equation 8) regarding the boundary conditions and determining the quantities of $\lambda$

- calculation of the velocities using the equations (4)

Figure 6 and 7 display the times at which the wind simulation has been done according to the times when the data of upper layer are present; in Tehran, these data are gathered at zero hour and 12 hours GMT.

Figure 6 shows the result of simulation at 0 GMT (3:30 local time). At this time, the intensity of the synoptic wind near the earth is less and the existing winds are the caused by the local mountain valley wind; as it can observe, the wind vectors are directed from the mountains towards the center of the city.

Figure 7 shows the result of simulation at 12 GMT. Although the power of the local wind system from valley to the mountain is greater than that from the mountain to the valley, there is no sign of the later due to the existence of powerful system caused by upper atmosphere.

In the next step the wind field outputs from MM5 model were ingested into the Mass Consistent model as an initial-guess wind field. In this case, the prognostic winds are interpolated to the fine-scale Mass Consistent model grid and the normal diagnostic adjustments for the fine-scale terrain are made. 


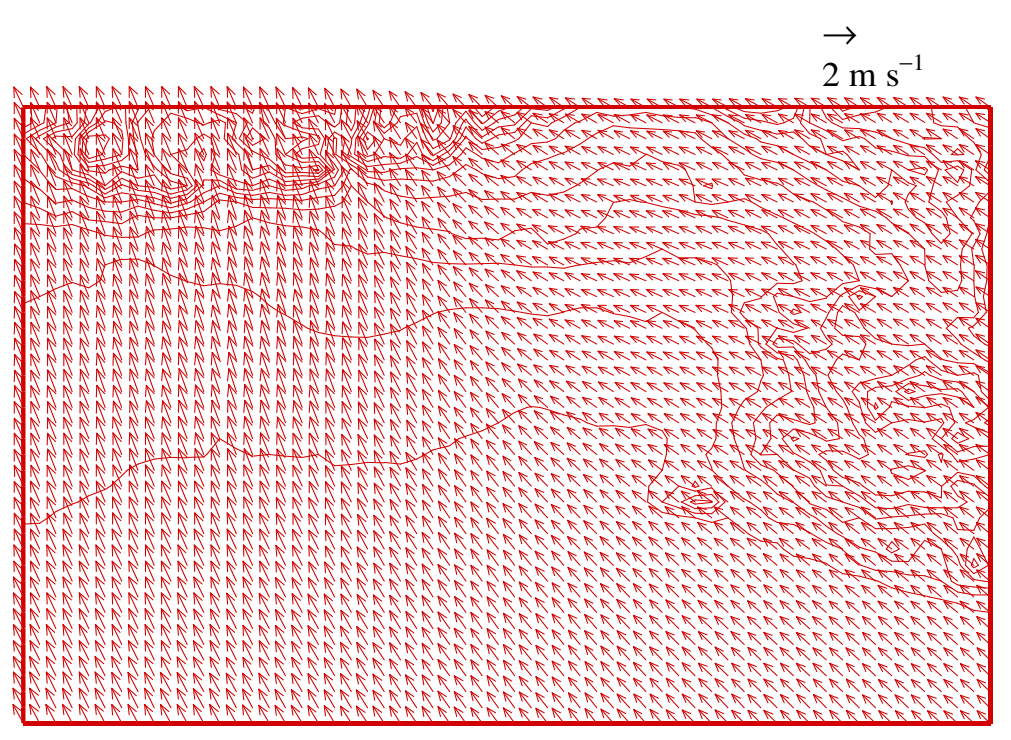

Fig. 7: Wind field simulation in Tehran on 2.9.2001 at 12 GMT at the height of 10 meters

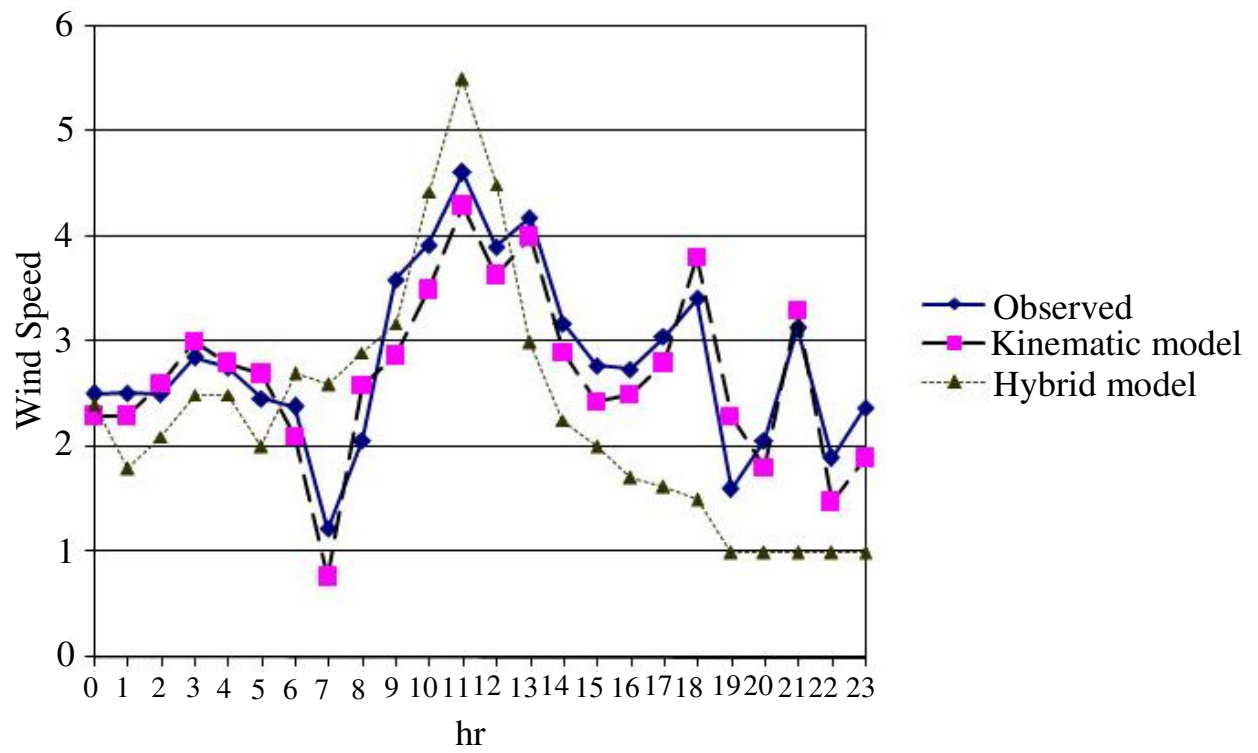

Fig. 8: Improvement of results using hybrid model

Finally, an objective analysis procedure is employed using all available observations. The Mass Consistent model utilized 14 layers in the vertical.

The Mass Consistent model utilized routine NWS observations with upper air radio sonde data (available every $12 \mathrm{~h}$ ) from stations and hourly surface data from surface stations. Figure 8 shows the improvement of results using Hybrid model (Combination of MM5 and model).

\section{CONCLUSION}

In this article, the wind field in Greater Tehran Area (GTA) is simulated by a diagnostic mass consistent model and based on the data gathered by meteorological towers installed in Tehran city and also the data from the upper layer atmosphere in Mehrabad Airport. The complex topography of Tehran has a great influence on the wind field of the city. As a result, regarding the subject of pollutant transformation and 


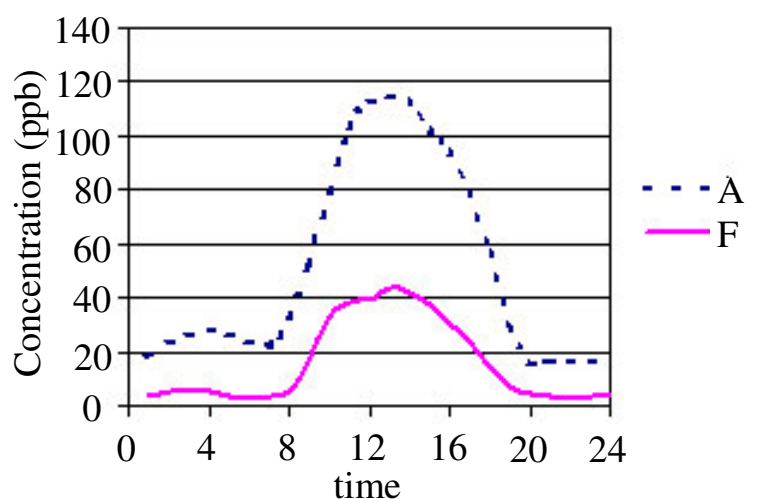

Fig. 9a: Concentration of carbon monoxide in two stations of fatemi and bazar during the summer 2003

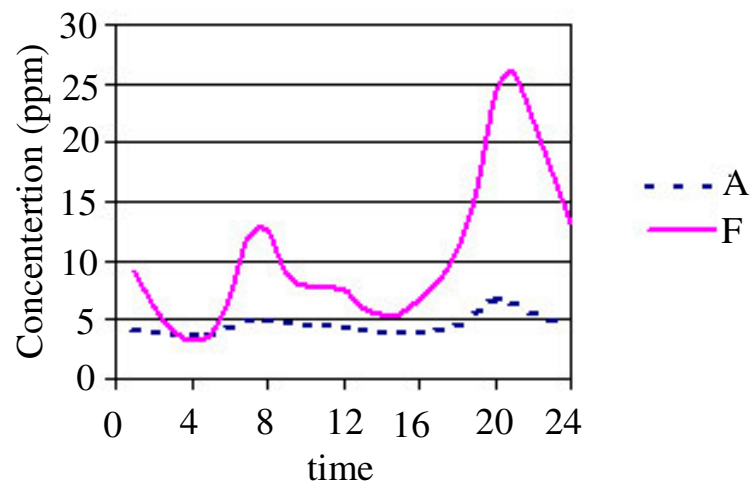

Fig. 9b: Compares the density of ozone in two stations of Fatemi and Bazar during the summer 2003

chemical reactions, knowing the pattern of wind field becomes even more essential. According to geographical and meteorological conditions of Tehran, the concentration of the pollutants differs from point to point, with regard to the season. In general, the concentration of the primary pollutants in the highly trafficked points exceeds the standard level, whereas in the down wind zones, the density of the secondary pollutants and ozone in especial is more than central zones. In addition to ozone, the other dangerous chemicals are produced by photochemical reactions such as PAN and other photochemical oxidants the effect of which on ones health is a lot more risky than the first pollutants. Therefore, recognizing the wind pattern is the most first parameter for surveying the mentioned phenomena. Figure $9 \mathrm{a}$ and $\mathrm{b}$, comparatively demonstrates the condition of two pollutants: Carbon Monoxide and Ozone, which are the primary and secondary pollutants respectively, in two points of Tehran city $($ Fatemi $=$ the highly trafficked central zone and Aqdaseye = downwind region). Obviously, in the central zones the concentration of the primary pollutants is greater than that of the fringe zones; concerning the second pollutants the situation is reversed.

The result of the wind field simulation reveals when the velocity of the synoptic scale wind is low, the condition of the wind flow is entirely affected by the local system of mountain-valley. During the day, the flow is towards the valley to the mountains, while at night it is from the mountain to the valley. The local systems of wind circulation such as mountain-valley and land-sea are closed systems that trigger in removing of the pollutants, their accumulation and their chemical changes in a definite area.

It should be noted that wind field simulation, by means of diagnostic models, depend entirely on the existing data and considering the time that one can not forecast wind field over the observed data. The results of wind field simulation using combination of diagnostic and prognostic models are significantly improved.

\section{Nomenclature:}

$\begin{array}{ll}D & =\text { wind turning angle } \\ E & =\text { constrain for wind field simulation } \\ f & =\text { Coriolis parameter } \\ f(r) & =\text { weighting function } \\ h & =\text { topography height } \\ H & =\text { top height for simulation } \\ J & =\text { combination of continuity eq. and } \\ & \text { constrain } \\ k & =\text { von karman constant }(0.4) \\ L & =\text { Monin Obukhov length } \\ r & =\text { distance between gird to station } \\ u & =\text { wind vector } \\ u, v, w & =\text { components of wind vector } \\ u_{0}, v_{0}, w_{0} & =\text { components of initial wind vector } \\ u_{*} & =\text { surface friction velocity } \\ x, y, z & =\text { Cartesian coordinates } \\ Z & =\text { vertical coordinates according to terrain } \\ & \text { following system } \\ z_{0} & =\text { roughness parameter } \\ z_{s l} & =\text { surface layer thickness } \\ z_{p b l} & =\text { planetary boundary layer heigh }\end{array}$




$$
\begin{array}{ll}
\alpha & =\text { correction ratio of horizontal an } \\
& \text { vertical directions } \\
\lambda & =\text { Lagrange multiplier } \\
\pi & =\text { difference between } H, h \\
\rho(z) & =\text { density function } \\
\omega & =\text { overrelaxtion parameter } \\
\Omega & =\text { specified area for wind field simulatio } \\
\psi_{m} & =\text { momentum similarity function } \\
\zeta & =\text { modified terrain following coordinate } \\
& \text { REFERENCES }
\end{array}
$$

\section{REFERENCES}

1. Sherman, C.A., 1978. A mass consistent model for wind held over complex terrain. J. Appl. Meteorol., 17: 312-319.

2. Beljaars, A.C.M., J.L. Walmsley and P.A. Taylor, 1987. A Mixed Spectral Finite Difference Model for Neutrally Stratified Boundary-Layer Flow over Roughness Change and Topography, BoundaryLayer Meteorol., 38: 273-303.

3. Xu, D. and P.A. Taylor, 1992. A non-linear extension of the mixed spectral finite difference model for neutrally stratified turbulent flow over topography, Boundary-Layer Meteorol., 59: 177-186.

4. Moussiopoulos, N., T. Flassak and G. Knittel, 1988. A refined diagnostic wind field model, Environ. Software, 3: 85-94.

5. Ross, D.G., I.N. Smith, P.C. Manins and D.G. Fox, 1988. Diagnostic wind field modeling for complex terrain: model development and testing. J. Appl. Meteorol., 27: 785-796.
6. Ratto, C. F., R. Festa, C. Romeo, O.A. Frumento and M. Galluzzi, 1994. Mass-consistent models for wind fields over complex terrain: The state of the art. Environ. Software, 9: 247-268.

7. Ludwig, F.L., J.M. Livingston and R.M. Endlich, 1991. Use of mass conservation and critical dividing streamline concepts for efficient objective analysis of winds in complex terrain. J. Appl. Meteor., 30: 1490-1499.

8. Moussiopoulos, N., T. Flassak, P. Sahm and D. Berkowitz, 1993. Simulation of wind field in Athens with the nonhydrostatic mesoscale model MEMO. Environ. Software, 8: 29-42.

9. Dudhia, J., 1993. A nonhydrostatic version of the Penn State-NCAR mesoscale model: validation tests and simulation of an Atlantic cyclone and cold front. Quart. J. R. Met. Soc., 121: 1493-1511.

10. Pielke, R.A., R.W. Cotton, R.L. Walko, C.J. Trcmback, W.A. Lyons, D.L. Grasso, M.E. Nicholls, M.D. Moran, D.A. Wesley, T.J. Lee and J.H. Copeland, 1992. A Comprehensive Meteorological modeling System-RAMS. Meteorol. Atmos. Phys., 49: 69-91.

11. Holstlag, A., 1984. Estimates of diabatic wind speed profile from near surface weather observations. Boundary Layer Meteor., 29: 3415-3159.

12. Van U. and A., Holstlag, 1985. Estimation of atmospheric boundary layer parameters for diffusion applications. J. Climate Appl. Meteor., 24: 1196-1207.

13. Zilitinkevich, S.S., 1972. On the determination of the height of the ekman boundary layer. Boundary Layer Meteor., 3: 141-145.

14. Panofsky, H.A. and J.A. Dutton, 1984. Atmospheric turbulence. Wiely New York. 